\title{
Albanian Parliament and Economic Concessions to Italy (1925-1928)
}

\author{
Bashkim Boseta \\ Ph.D candidate. Facoltà della Storia e Filologia,Universita di Tirana Albania \\ Email:bashkimboseta@yahoo.it
}

\section{Doi:10.5901/mjss.2013.v4n10p169}

\begin{abstract}
This work will address the following issues: 1-Discussions in Albanian Parliament about the economic situation in the country. Stand of Albanian Parliament on political-economic orientation of Albanian Government regarding Italy. Views of Albanian Parliament about Italian political system and experience of Italian administration in relation to further organization of Albanian state and economy. 2- Views and different groupings at Albanian Parliament and their stances regarding Italian-Albanian agreements for establishment of National Bank of Albania, loan from Italian company S.V.E.A and other economic concessions. 3- Stand of Albanian Parliament about level of practical efficiency of Italian-Albanian agreements and other economic concessions, problems occurred during their implementation and Parliament's approach about these problems.
\end{abstract}

L'Albania e l'Italia sono due popoli che hanno avuto contatti e legami storici remoti, i quali sono rafforzati durante il periodo di Scanderbeg e dalla comunità arberesh collocata in Italia dopo l'invasione turca dell'Albania. Dopo la creazione dello stato italiano fu evidente un ripristino dell'attenzione italiana verso l'Albania ai tempi del Presidente del Consiglio dei Ministri Francesco Crispi, il quale fu di origine albanese. L'Italia insieme all'Austro-Ungheria ha avuto un ruolo importante nella creazione dello Stato albanese. L'Italia fu tra I primi paesi che ha riconosciuto lo stato indipendente albanese. Con l’arrivo al potere di Benito Mussolini nel 1922, cominciò a suscitare interesse per intervenire nei Balcani e l'Albania era considerata come punto di sostegno e di passaggio. Negli anni 1920-24 in Albania ebbero luogo avvenimenti importanti dove si notano i tentativi per il consolidamento dello stato nel piano interno ed esterno. II 24 dicembre 1924 Ahmet Zogu arriva al potere, viene proclamata la Repubblica Albanese e A. Zogu il suo Presidente. Dopo aver messo sotto controllo la situazione interna, l'attenzione passò sulla grave situazione finanziaria e l’assicurazione di una alleanza esterna con un grande potere. A. Zogu, "giunse alla conclusione che era appunto I'Italia quella che si doveva far attirare verso l'economia albanese. L'Italia era uno stato economicamente potente, oltre a questo, non aveva confini terrestri diretti con l'Albania". ( La Storia del Popolo Albanese, volume III, 2007).

In questo punto influenzò da un lato anche la diplomazia inglese e il rischio che poteva correre dalla Jugoslavia dall'altro lato, poiché l'Albania tradizionalmente era stata alla mira dello smembramento territoriale dai paesi vicini. "Per questi motivi, il 30 gennaio 1925, A. Zogu si rivolse a B. Mussolini, Capo del Governo italiano del tempo, e chiese a lui di stabilire rapporti amichevoli tra Roma e Tirana." ( Duka, 2007, p. 161).

II Parlamento albanese negli anni 1925-1928 era composto da due Camere: la Camera dei Deputati e il Senato. "In questo modo, in Albania, per la prima e l'ultima volta si istituiva il sistema bicamerale sulla base della parità dei poteri di entrambi le Camere. ( Anastasi, 2007, p. 75 ). II Parlamento di questi anni funzionò senza l'opposizione, siccome gli avversari di A. Zogu dopo il suo ritorno al potere se ne erano andati all'estero. Tuttavia il Parlamento albanese di questi anni ebbe un intensa attività, dove una parte importante di essa fu occupata dalla discussione e dall'approvazione delle concessioni economiche con l'Italia.

La questione piu importante che impegnò l`attezione del governo di Zogu durante l’anno 1925 era la fondazione di una Banca Nazionale e l'assicurazione di un mutuo per lo sviluppo del paese. ( Swire, 2005 p.360) Pretendente per la banca era il gruppo inglese Midland Bank. Però il governo italiano non poteva permettere questa cosa, poiché non raggiungerebbe lo scopo per intervenire in Albania. In queste circostanze cominciò la corrispodenza diplomatica tra Roma e Londra, per la questione della precedenza italiana nell'economia albanese. "Londra, la quale subito dopo la Prima Guerra Mondiale aveva dato il consenso al capitale italiano per avere una posizione speciale in Albania, rinunciò al proprio piano ed ha aperto la strada all' Italia in Albania." ( La Storia della Banca Centrale in Albania. 2003, p.18). La convenzione per la fondazione della Banca di Albania fu sottoscritta il 15 marzo 1925 a Tirana tra Myfit Libohova, Ministro delle Finanze e viceministro degli Affari Esteri albanesi e Mario Alberti, rappresentante del Gruppo Finanziario Italiano e Presidente della Banca Credito Italiano. II capitale nominale sarebbe 12.5 milioni di franchi d'oro. Secondo 「'accordo, gli albanesi avrebbero in possesso 49\% del capitale azionario. Nel Gruppo Finanziario italiano presieduto da 
Mario Alberti, partecipavano le tre banche più potenti d'Italia: Credito Italiano, Banca Commerciale Italiana, Banca Nazionale di Credito, Banca di Roma insieme a quattro piccole banche italiane (Frasheri, 2011, p.97).

Oltre agli italiani possedevano azioni anche alcune banche europee da Svizzera, Belgio e Jugoslavia. Secondo 「accordo, la direzione della Banca sarebbe composta da due albanesi e due italiani. II presidente sarebbe italiano e goderebbe il voto definitivo. Presidente fu eletto M.Alberto, mentre consigliere delegato Andrea Gambino. La Banca godeva il diritto di emissione delle banconote ed il conio delle monete metaliche e divisionali. II 15 marzo ancora fu fondata la Società per lo Sviluppo Economico dell'Albania, altrimenti nota come S.V.E.A. Cio`assicurerebbe all'Albania un mutuo su 50 milioni di franchi d'oro con un interesse annuale di $7.5 \%$ e ad un termine di estinzione per un periodo di 40 anni. "in base all accordo il mutuo sarebbe usato per eseguire i lavori pubblici in Albania, per la costruzione dei canali, dei ponti, delle strade ed il miglioramento delle terre agricole e lo sviluppo dellagricoltura, per la costruzione delle ferrovie ( Fishta, 1971, p.43).

Al Parlamento albanese la questione della Banca e della S.V.E.A fu discussa largamente. Durante le discussioni al Parlamento riguardo la banca e il mutuo della S.V.E.A dà nell'occhio il consenso degli accordi stipulati con l'Italia. Nelle tredicesima riunione, il 23 giugno 1925 fu presentato il rapporto delle commissioni delle finanze e dell'economia da Hiqmet Delvina, dove si dà la conclusione delle commissioni che: "Le condizoni dell'accordo rispetto alla Banca ed al mutuo sono ragionevoli ed utili per lo Stato albanese, perciò devono essere acetate." (Discorsi della Camera dei Deputati, 1925, pp.101 102). Durante la presente riunione furono approvate anche le garanzie che il Governo albanese offriva per il mutuo, le quali erano: il gettito delle tasse dalle dogane di 6 milioni di franchi d’oro, i monopoli dello Stato sul sale, le carte da sigaretta e i fiammiferi di un valore di 2,5 milioni di franchi d’oro. Nelle disscussioni dei deputati furono messe in rilievo anche i vantaggi che approfitava I'Albania da quest'accordo. II deputato Xhaferr Ypi all'inizio critica la borghesia albanese, i governi precedenti, che secondo lui, non hanno avuto il coraggio per fondare una banca e poi elenca i vantaggi della Banca e del mutuo, i quail erano: un mutuo di 50.000 .000 franchi d'oro, in modo che con questo denaro potremmo affrontare la costruzione delle strade, dei ponti, con questo denaro potremmo stampare le banconote dell'Albania, le quali circolando pure all'estero con l'immagine del Capo della Repubblica e menzionando anche il nome dell'Albania ci farà conoscere al mondo. L'agricoltore, presentando la propria merce come garanzia, potrà avere del denaro dalla Banca di un interesse molto basso da quel che c'è nella piazza di oggi. "Quindi, tenendo presente tutti questi vantaggi che avremmo mi sacrifico ogni danno che ci puo' correrre, il quale sara' minimo e con gioia l'accetto e do il mio voto." ( Discorsi della Camera dei Deputati, 1925, p.166).

I deputati albanesi non solo che evidenziarono i lati positivi e i vantaggi che avrebbe l'Albania dalla convenzione con I' Italia, ma hanno cercato di giustificare anche le ragioni del mantenimento della maggioranza delle azioni dalla parte dei dirigenti italiani. Cosi, il relatore della commissione parlamentare che aveva esaminato la convenzione con I'Italia il sig. Vasil Bamiha si è espresso: "Le azioni fondamentali saranno tenute dai fondatori della Banca, ma, non pensate signori che questi ( $\mathrm{i}$ fondatori) avranno un privileggio speciale; perché anche la legge commerciale italiana non gli dà nesssun privileggio economico, solo che avranno la maggioranza nellamministrazione della Banca, cioè per questo scopo sono create queste azioni. Ed è giusto siccome possiedono la maggioranza del capitale. $51 \%$ l'avranno i fondatori della banca ( gli azionisti) e $49 \%$ il popolo albanese. La presente Banca sarà gestita da un consiglio di sei persone: tre italiani e tre albanesi." ( Discorsi della Camera dei Deputati, 1925, p.167).

Benche avesse grande consenso per gli accordi con l'italia, ci furono anche delle voci particolari dei deputati, i quali cercarono un controllo del Parlamento sul governo per il modo in cui si gestirebbero i soldi offerti dal mutuo dell'Italia. Cosi, il sig. Milto Tutulani insieme ad altri deputati hanno cercato la creazione di una commissione parlamentare permanente allo scopo di controllare il lavoro del governo. Lui dichiarò: "Noi proponiamo che si formi dalla Camera una commissione per un controllo parlamentare permanente, il quale sarà incaricato a controllare ogni attività del governo sulla buona applicazione delle condizioni di queste convinzioni e dello spendere del muto, soltanto per I lavori che vengono assegnati in quella convenzione." (Discorsi della Camera dei Deputati, 1925, p.97).

Ciò ha incontrato l'opposizione dei rappresentanti del governo di M.Libohova, il quale accusò M.Tutulani che voleva mettere sotto controllo la Banca e il Gruppo Finanziario italiano. Dopo la proposta di M. Tutulani fu messa in processo di voto e non ha vinto. II tempo ha dimostrato che M.Tutulani aveva avuto retta, perché più tardi sono venuti fuori gli abusi di M.Libohova. La legge organica della Banca Albanese fu approvata dal Parlamento albanese il 23 giugno 1925. II 5 luglio 1925 invece, fu approvata al Senato la legge, che permetteva alla Banca Nazionale Albanese il diritto esclusivo per l'emissione delle banconote, e per il conio delle monete d’oro e di argento.

Con l'accordo raggiunto sulle azioni della banca, sebbene gli azionisti stranieri avessero avuto il $51 \%$ delle azioni, il Gruppo Italiano rischierebbe la maggioranza, perché c'erano anche delle banche azioniste straniere, e gli albanesi, secondo la convenzione avrebbero $49 \%$ delle azioni. Allora il Gruppo Finanziario Italiano ha agito. Alessandro Roselli nel 
suo libro Italia e Albania dice che da parta italania occoreva perciò abbassare di fatto il $49 \%$ che la convenzione riservava agli albanesi. II 30 \% del capitale doveva essere sottoscritto da albanesi residenti in Italia. Lui diceva: "Quanto al restante $19 \%$, poiché all ultimo giorno consentito per la sottoscrizione nessun albanese aveva proceduto, anche questa quota fu assunta dal gruppo italiano. Minime assegnazioni furono fatte successivamente a funzionari albanesi, senza turbare il preesistente equilibrio." (Roselli, 1986, p. 65). Per le loro azioni gli italiani hanno trovato l’appoggio di M.Libohova, Ministro delle Finanze Albanesi, autore della convenzione con I'Italia in cambio delle grosse somme monetarie prese sottomano. Lui, da una parte, ha tenuto nascosta la data della scadenza del termine degli acquisti delle azioni dagli albanesi interessati, e cosi ha dato l'opportunità al Gruppo Finanziario Italiano di fare acquisti anche dall altra parte: sono fatte degli acuisti di alcune azioni dagli azionisti albanesi. (Ajet Libohova, Neshat Pashë Vlora, Eqerem Vlora) i quali molto presto hanno trasferito queste azioni agli italiani. Di conseguenza il Gruppo Fianziario italiano ha avuto la somma assoluta delle azioni della Banca Nazionale Albanese. Questa questione fu trattata largamente al Parlamento Albanese in alcune delle sue riunioni. M. Libohova fu accusato di corruzione a danno dello Stato albanese. Per indagare questo problema, il 24 ottobre 1925, fu creata una commissione d'inchiesta parlamentare, la quale ha evidenziato tutte le infranzioni commesse da lui. Secondo le inchieste fatte dalla commissione risultò che M. Libohova aveva commesso gravi infrazioni. Lui fu accusato per il deposito del mutuo di 1.000 .000 franchi d'oro in lire italiane ricevuti dal Gruppo italiano causando alla cassa dello stato un danno di 60.744,09 franchi d'oro. Aveva comprato due vapori all insaputa del governo, mentre le finanze erano in difficoltà ecc. L’accusa più grave che la commissione fece a M.Libohova fu che aveva tenuto nascosto per gli albanesi il termine della sottoscrizione delle azioni della Banca che scadeva il 5 maggio 1925. Di conseguenza gli albanesi persero l'opportunità della sottoscrizione delle azioni a loro appartenenti in base allarticolo 4 della convenzione della Banca Nazionale Albanese. Con questi atti M.Libohova aveva offerto i diritti dell'Albania agli stranieri" si concludeva il rapporto della commissione. (Rapporto della commissione d inchiesta parlamentare, novembre 1925, p 30-32) M. Libohova si era allontanato dal Presidente della Repubblica sin dal settembre dalle funzioni governali, scappando pure dal processo giudiziario visto che esisteva la possibilità dell annullamento della convenzione del 15 marzo.

La situazione dei rapporti tra la Banca e il Parlamento Albanese non fu sempre di pieno consenso. Cosi a maggio dell'anno 1927 al Parlamento ebbero luogo aspre discussioni tra I deputati, dove la banca si accusava che non concedeva crediti agli albanesi, emettteva l'oro all'estero e coniava delle monete metaliche, che non era il diritto della banca, ma dello Stato Albanese. Quindi, nel suo discorso Xh.Ypi diceva che la nostra Banca Nazionale, anche quando concede a qualche commerciante qualche soldo glieli concede con molti ostacoli e difficoltà. La Banca non sta cambiando la vcaluta con l'oro, e in questo modo, ha tolto il valore alla valuta. Quando si rivolge il mondo per il cambio degli assegni risponde che nell'oro ci sarà uno sconto di meno e con la banconota albanese di piu. Lui dichiarava: "II conio delle monette metaliche. La banca ha emmesso alcuni ferri di cavallo assegnando un prezzo di 5 franchi d'oro perché quel denaro con quel peso vale 5 corone. La moneta metalica è un diritto dello Stato e non della Banca" (Discorsi del Parlamento 1927 pp 387- 388). In oltre si accusa il Ministero delle Finanze, visto che non esegue i controlli sulla banconota emmessa in circolazione: sono privi di data, privi di numero, privi di serie. La banca fu protteta dal Ministro delle Finanze Fejzi Alizoti, il quale dichiarò: "Secondo la convenzione la Banca non ha l'obbligo di aiutare gli agricoltori." Sebbene venga chiamata Banca Nazionale, il denaro è straniero, per la questione delloro, secondo lui, nessuno è andato alla Banca a cambiare le banconote con l'oro. Per le monete gode il diritto del conio ma devono ottenere il permesso dal governo e II Ministero delle Finanze ha vietato la moneta di 5 franchi, di 2 franchi, abbiamo accettato solo denaro in nickel (Discorsi del Parlamento 1927, p.388.). Nell anno 1928 al Parlamento fu discusso il moratorium con S.V.E.A per rimandare il credito che apparteneva allo Stato albanese. Per prima, I deputati chiesero informazioni per il monatorium. Dopo esser data l'informazione il presidente della commissione di finanze del parlamento F.Alizoti, Ministro delle Finanze dichiara che rimandando il termine del pagamento del credito noi abbiamo interesse perché approfitiamo molto dai lavori pubblici. Questo progetto di legge fu messo in votazione il 2 aprile 1928 nella tredicesima riunione e fu approvato.

Oltre alla convenzione della Banca ed il mutuo al Parlamento albanese degli anni '25-28 furono discusse anche le altre concessioni con l'Italia, le quali riguardavano a molti campi. Uno di essi fu il Trattato del Commercio e della Navigazione tra I'Albania e l'Italia, in cui l'Italia prese lo statuto della nazione piu favorita: "Il 20 gennaio 1924 tra I'Albania e l'Italia fu sottoscritto un patto segreto marino-commerciale, che riconosceva all' Italia lo statuto della nazione piu favorita " (Fischer 2004, p.99.) Questa convenzione con il ritorno di A. Zogu al potere e il suo avvicinamento ulteriore all Italia doveva diventare realtà. Di conseguenza il protocollo passò al Parlamento per la ratifica. Dopo molte discussioni fu deciso che il trattato venisse tradotto di nuovo perchè non si capiva dalla brutta traduzione. Nella ventitresima riunione il 14 luglio 1925 il Trattato del Commercio e della Navigazione con I'Italia si e` messo dinnuovo in discussione. II Trattato, 
dalla maggioranza dei deputati fu sostenuto e si considerò utile per l'Albania. Alcuni deputati nelle loro discussioni se ne accorsero che il trattato era contrario al Decreto Legge presentato dal governo sul divieto del transito. In questo punto ci furono scontri in discussioni tra deputati e il rappresentante del governo sig. M. Libohova, il quale insistette di abrogare la legge che non consentiva il transito. Oltre a questi, ci furono dei deputati che contestarono il Trattato, perché lo ritenevano dannoso dal punto di vista economico per lo Stato Albanese. Qui si distinse il sig. Ferid Vokopola, il quale nel suo discorso disse: "Come sapete Vostri Signori, dal punto di visto economico vince sempre la nazione che è più prosperosa e non noi che non abbiamo un bilancio equilibrato, che non possediamo una tariffa doganale studiata sulle basi tecniche e con questo trattato leghiamo il paese mani e piedi e non potremo prendere i dovuti provvedimenti che ci servono per migliorare la nostra situazione. lo, dal punto di vista economico lo ritengo dannoso questo trattato senza essere studiati in modo approfondito i punti che ho elencato di sopra. E non credo che quei due anessi rispondono ai bisogni vitali dello Stato". ( Discorsi della Camera dei deputati, 1925, p.407. ) Tuttavia ciò non fu preso in considerazione dalla maggioranza dei deputati e fu deciso di modificare la legge sul transito in funzione al trattato con l'Italia.

L’interesse per il petrolio albanese si rese vivo specialmente subito dopo la Prima Guerra Mondiale. Mostrarono interesse gli stati come Inghilterra, gli Stati Uniti d’America, I'Italia e la Francia. Gli scontri maggiori furono tra I'Italia e l'Inghilterra perché la società britannica anglo-persiana aveva preso concessioni di petrolio in Albania sin dal 1921 ed inoltre questa società aveva avuto la promessa di A. Zogu che avrebbe avuto campo libero nella sua attività. Cosi dal piano di nove punti che Contarini, il Segretario Generale del Ministero degli Esteri dell' Italia presantò ad A. Zogu, questo l'ultimo non accettò solo la richiesta per dare in concessione per la ricerca e lo sfruttamento dei campi di petrolio in Albania perché il Zogu si era impegnato con priorità alla società anglo-persiana, la quale finanziando con 25.000 sterlina per il rovescio del Governo di Fan S, Noli, aveva assicurato prima la promessa per la concessione del petrolio in Albania. ( Fishta, L 'intervento del capitale straniero e le sue conseguenze schiaviste per l'Albania. 1979, p.53) La diplomazia inglese e quella italiana dopo alcune trattative, raggiunsero il consenso e cosi fu aperta la strada all' Italia per chiedere le concessioni del petrolio in Albania.

Al Parlamento Albanese, il trattamento delle concessioni del petrolio con l'Italia prese un posto rilevante. La prima concenssione discussa ed approvata fu quello tra il Governo Albanese e le Ferrovie dello Stato Italiano con rappresentante Ugo Piloti. Per questa convenzione al parlamento ci furono pochi dibattiti e quelli casomai la concessione avesse qualche differenza con quella della società anglo -persiana e pure la soluzione del problema da parte del governo con una società francese, la quale aveva presentato domanda per lo stesso territorio. Dalle discussioni risulta che la convenzione è la stessa con quella della società anglo - persiana, soltanto con una differenza che la parte italiana costruirebbe in Albania una raffineria di petrolio. II sig. Rauf Fico chiede chiarimenti rispetto all'articolo 19 della convenzione dove la società per i suoi mezzi si diceva che era libera da ogni tassa doganale e lui chiede se tutto cio vale anche per le tasse del municipio. Per questo, la risposta di Kostaq Kote, Ministro dei Lavori Pubblici è che sarà risolto dall'arbitraggio. La discussione fu chiusa da Selaudin Blloshmi, il quale dice: "Siccome il governo questo punto l'ha incluso in arbitraggio, ogni soluzione o interpretazione che noi diamo sembra che sia inutile, poiché la decisione data dall 'arbitraggio per gli anglo -persiani varrà anche per le altre società." ( Discorsi della Camera dei deputati, 1925, p.296). Poi viene messo in votazione ed il contratto si approva in modo unanime il 10 luglio 1925. Secondo il contratto la concessione durerebbe fino al 1985, poi l'investimento passerebbe allo Stato Albanese.

All inizio del marzo 1926 la Società italiana presentò una richiesta per altri 25 mila ettari da sfruttare, la quale fu approvata e il governo prendeva la responsabilità dell’applicazione della legge di espropriazione, casomai I proprietari dei terreni non fossero d'accordo. Durante questa riunione ci furono delle discussioni sugli specialisti che doveva portare la società italiana in Albania e si stabilì, anche se ci furono delle contestazioni, che la società portasse in Albania soltanto specialisti per quanto riguardo al petrolio. II Senato intervenne per la modificazione fatta dalla Camera dei deputati al termine linguistico "per quanto riguarda al petrolio". Secondo il Senato se venisse aggiunta una tale condizione solo per una concenssione in specifica, si danneggerebbe il morale, la dignità e la credibilità della società concessionaria. Le modifiche fatte dal Senato erano: La concenssionaria gode il diritto di mettere in uso I terreni privati necessari, in case di non mettersi d`accordo con il proprietaro della terra sul valore stabilito, è obbligato entro 10 giorni dal giorno dell'occupazione delle terre, per rivolgersi all'amministrazione locale per attivare gli atti della legge di espropriazione, Articolo nr.16. A questo articolo viene aggiunto un altro paragrafo in questo modo: "Per tutelare la salute degli impiegati e degli operai la concessionaria è obbligata ad assicurare i medici necessari ed a distribuire i farmaci gratis (Discorsi della Camera dei deputati, 1926, p.346).

Ma non sempre le delibere del Senato venivano approvate in modo unanime. Anche questa delibera ebbe molti sostenitori, ma ebbe anche contestazioni. Cosi, dr.Simonidhi dopo aver elencato il buon lavoro della commissione, si mette apertamente contro il Senato. Lui dichiara: "Io sono contro questa delibera ed insisto sulla delibera precedente, 
oppure dobbiamo dare al vocabolo la giusta interpretazione dato che questa parola ha una larga interpretazione. II Senato dice che questo si fa solo per la Ferrovia dello Stato, però io dico che sia alla concessionaria di Puka, sia a quella di Memaliaj, sia in ogni altra concessionaria, lo Stato deve assicurare che gli impiegati e gli operai albanesi vengano curati dai medici albanesi". ( Discorsi della Camera dei deputati, 1926, pp. 347-348). Dopo molte disscusioni fu deciso di formarsi un gruppo di quattro deputati e due dal Senato per discutere il problema. II 21 aprile 1926 nella venticinquesima riunione le modifiche del Senato passano attraverso il voto e vanno approvate. Una altra concessione che fu discussa al Parlamento fu quella tra il Governo albanese e la Società italiana delle Miniere di Selenica rappresentata dall'ing. Giovanni Nobili. Questa convenzione fu discussa il 10 luglio 1925. Secondo cui la società prenderebbe una superfice di 2140 ettari, con il diritto da sfruttamento di 800 ettari e termine d’uso di 50 anni. Questa convenzione fu approvata con i voti unanimi il 11 luglio 1925. II Parlamento anche nel caso di questa società fu disposto ad approvare le modificazioni richieste dal governo all interesse della società italiana. Cosi, ad aprile 1928 fu approvata la richiesta della società che chiedeva rimandare il termine delle ricerche per due altri anni ancora facendo i cambiamenti della prima convenzione. " Art.1 Alla convenzione di petrolio stipulata tra il Governo albanese e la Società delle Miniere di Selenica con la sede a Roma, Piazza S.S. Apostoli 81, art. nr.2. - Viene aggiunta questa disposizione: II termine di tre anni stabilito nell'articolo 2 della convenzione, come periodo di ricerca, continuerà anche per un altro termine di due altri anni cominciando dal 2 maggio 1928". (Discorsi del Parlamento, 1928, p. 84.)

La concessione successiva con I'Italia che fu discussa al Parlamento fu quello con la società Sindicato Italiano Giaccimenti Minerari Albanesi sullo sfruttamento del carbone di Memaliaj. L’unica discussione fu dal sig. R. Fico, il quale dichiarò: "lo vorrei per prima sapere il potenziale del carbone, quanto si trova e dove si trova. Senza avere spiegazioni e senza sapere quanto carbone si trova in Albania, che cosa stiamo votando?" (Discorsi della Camera dei deputati, 1925, p. 848.) Questo contratto fu accettato in principio il 2 novembre 1925. Anche in questo punto intervenne ancora il Senato , il quale modificò la concessione del carbone di Memaliaj. Nell Articolo nr. 6, punto $\mathrm{c}$, si diceva che la società deve pagare, secondo gli articoli della legge viggente delle miniere, una tassa proporzionata sul prodotto. II Senato ha considerato piacevole che la tassa menzionata di sopra cambiasse in $4 \%$ sulla produzione del carbone brutto. Questa tassa verrà pagata ogni tre mesi in base al prezzo medio togliendo le spese necessarie dello trasporto del carbone dal porto albanese al porto di vendita. (Discorsi della Camera dei deputati, 1926, p.197-198). Dopo questa modificazione i deputati lodano il lavoro del Senato. II rappresentante del governo il sig. Musa Juka dichiara che sia il Governo Albanese, sia la società italiana sono d’accordo con le modificazioni fatte e cosi loro si accettano e si approvano.

Nel Parlamento Albanese, nella maggior parte dei casi, alcuni deputati albanesi sono stati grati nei confronti d'Italia e hanno lodato il Governo italiano e il regime fascista. Ci sono stati dei casi in cui nelle loro discussioni alcuni deputati accusavano gli imprenditori albanesi e lodavano quelli italiani. Nel 1926 quando si discuteva per la sistemazione di due caserme militari a Tirana e a Durrazzo si è dubitato per corruzione dei fondi ma sono esclusi gli italiani. Cosi, Bahri Begolli dichiarava: "Io per gli organizzatori italiani non ho nessun dubbio e ho rispetto per loro e tutto il mondo lo sa che io ho rispettato gli italiani e li rispetto sempre, però, io sul Comandante Generale non ho fiducia. (Discorsi della Camera dei deputati, 1926, p.568) In questo caso il comandante era albanese. Anche nei momenti quando fu criticata la Banca oppure quando ci furono dei problemi con S.V.E.A non fu mai attaccato lo Stato italiano. Alcuni deputati nelle loro discussioni lodarono il regime fascista. Cosi Javer Hurshid, durante un discorso tenuto in Parlamento dichiarava: "Io sarei molto felice se l'Albania potesse avere un'organizzazione politica come il fascismo, visto che soffre per la mancanza dell'ordine, per il potere legale e leggitima del popolo. (Discorsi della Camera dei deputati, 1925, pp.11571158).

Le convezioni economiche tra I'Italia e l'Albania e l'atteggiamento che ebbe il parlamento verso loro, sono rassegnate anche nella stampa albanese. Cosi, ci furono dei giornali che sin dall inizio erano contro gli accordi economico con l'Italia. Esse, nei loro scritti, mostravano i lati negativi degli accordi per il popolo albanese. Cosi, il Giornale Repubblica nel suo dodicesimo numero, il 21 aprile 1925, scriveva: "Si danno concessioni, trattati e differenti convenzioni, dalle quali alcune non sono solo dannose, ma anche pericolose per la nostra nazione e per il nostro Stato. Soprattutto la concessione del mutuo dello Stato con la Banca di emissione è un impiego tanto brutto e da piangere, quanto da non credere, se non si vede e non si legge il contratto concessionario". (Repubblica, 1925, p.1). Aspre critiche contro gli accordi fecero i giornali pubblicati fuori dall'Albania che erano in opposizione al regime di A. Zogu, come: L'Albania Nuova, La Libertà Nazionale, ecc.) Una parte della stampa albanese che all inizio erano pro gli accordi, però dagli anni 1926 ed in poi, quando cominciarono ad apparire i primi problemi con la Banca e la S.V.E.A per varie questioni iniziarono anche le critiche per loro. Cosi il Giornale Telegraf, in uno scritto di Koste Çekrezi, nel suo numero 80, il 16 novembre 1926 scriveva: "Cosi come stanno gli affari in Europa e nei Balcani la nostra relazione con l'Italia ci da la piena sicurezza per il nostro essere nazionale. Purtroppo, io mi accorgo che nello sviluppo dei rapporti italo-albanesi si notano 
due correnti. II corrente politico rappresentato dal Governo italiano e il secondo dalla Banca e la S.V.E.A. Da questi gruppi, quello più forte e più dannoso per l’amicizia e il legame tra i due stati e` quello, i cui interessi si rappresentano insieme dalla S.V.E.A e la Banca Nazionale dell'Albania. S.V.E.A ci ha dato e non ci ha dato un prestito di 50 milioni di franchi d'oro verso al quale il popolo albanese si obbliga a restituire 70.500 .000 franchi d'oro oltre all interesse che verrà pagato annualmente. Dalla sua parte "la Banca Nazionale di Albania" sta raccogliendo l'oro del popolo albanese e lo sta portando a Roma, lasciandoci in mano delle banconote prive di date, nprive della firma di qualche ufficiale albanese, e prive di qualche garanzia che più tardi potrebbero essere scambiate con l’oro qui in Albania ( Telegraf, 1926, p.2). Dall altra parte ci furono dei giornali pro agli accordi con l'Italia e sostenevano in continuazione il lavoro della Banca e la S.V.E.A in Albania. Qui si distingue il Giornale Albanese e tutti gli altri giornali pro il governo. Cosi, il Giornale Albanese nel suo numero 7 , il 17 luglio 1927 scriveva: "Accanto alla regolamentazione finanziaria tra i problemi più gravi senza dubbio veniva di seguito la regolamentazione economica, e il presente compito fu preso dalla società S.V.E.A , la quale é completamente libera nele sue azioni dalla Banca Nazionale. La società, dopo un appoggio benevole del Governo Italiano e del Governo Albanese, nel marzo del 1925 diede un mutuo di 50 milioni di franchi d’oro per fini economici e amministrativi. La società ha solo l'incarico per finanziare i lavori pubblici fatti in Albania, senza chiedere nessuna delle garanzie pesanti, le quali mettono le azioni sotto la sorveglianza delle società straniere ( Giornale Albanese, 1927, p.1).

In conclusione si può dire: In primo luogo, il Parlamento albanese negli anni $1925-1928$, partendo anche dalla difficile situazione economica del paese, ha dato un ampio spazio alla discussione e all'approvazione delle convenzioni economiche con l'Italia. Le presenti convenzioni erano numerose e comprendevano quasi tutti i campi dell'economia.In secondo luogo, il Parlamemto albanese con l'approvazione di presenti convenzioni contribuì allo sviluppo economico dell'Albania, visto che fu fondata la Banca Nazionale, anche se con il capitale straniero, entrò in circolazione la moneta nazionale, sono fatte delle strade, costruzioni ecc. Con la convenzione per il petrolio, per il carbone, abbiamo gli esordii dello sviluppo industriale del paese.In terzo luogo, il Parlamento albanese, escluse voci particolari e in casi molto rari, non volle oppure non riuscì a capire che le presenti convenzioni anche se aiutarono l'economia albanese, erano in funzione della politica del governo fascista italiano, la quale mirava l'espansione dei suoi territori, il dominio e l'invasione dell'Albania, e di altri territori. II tempo ha mostrato che questa era la verità.

\section{References}

Anastasi, A. 2007. La Storia del Diritto Costitutivo in Albania 1912- 1939. Pegi.

I Discorsi della Camera dei Deputati (1925). Tirana.

I Discorsi della Camera dei Deputati( 1926) . Tirana

I Discorsi del Parlamento (1927). Tirana.

I Discorsi del Parlamento (1928). Tirana.

La Camera dei Deputati, ( novembre 1925) II rapporto della commmissione d’inchiesta parlamentare sulle azioni di sig. M.Libohova, ex. Ministro delle Finanze e vice Ministro degli Affari Esteri. 30-32.

Duka. V. (2007) La Storia dell'Albania 1912 - 2000. Tirana: Kristalina-KH.

Fischer, B. J. ( 2004) II Re Zog e lo sforzo per la stabilità in Albania. Tirana : Çabej.

Fishta, I. (1971) Il sistema monetario e del credito in Albania 1925-1944. Tirana: Tipografia Mihal Duri.

Fishta, I. ( 1979) L intervento del capitale straniero e le sue conseguenze schiaviste per l`Albania. Tirana : Accademia delle Scienze della Repubblica Popolare Socialista dell'Albania, I'Istituto degli Studi Economici.

Frasheri, K. (2011). La moneta, il credito e la banca in Albania nel corso dei secoli. Tirana : Toena.

Giornale Albanese, (17 luglio 1927). p.1.

La Storia della Banca Centrale in Albania. ( 2003). Tirana: La Tipografia della Banca dell'Albania.

La Storia del popolo albanese volume III. (2007.) Tirana: Toena.

Reppubblica. (21 aprile1925) . p.1.

Roselli, A. (1986). Italia e Albania: Relazioni finanziarie nel ventennio fascista. Bologna: II Mulino.

Swire, J. (2005) L’Albania, la Formazione di un regno. Tirana: Dituria.

Telegraf. (16 novembre1926) . p.2. 